\title{
ON A REGULARITY THEOREM FOR WEAK SOLUTIONS TO TRANSMISSION PROBLEMS WITH INTERNAL LIPSCHITZ BOUNDARIES
}

\author{
L. ESCAURIAZA, E. B. FABES, AND G. VERCHOTA
}

(Communicated by Barbara L. Keyfitz)

\begin{abstract}
We show that if $u$ is a weak solution to $\operatorname{div}(A \nabla u)=0$ on an open set $\Omega$ containing a Lipschitz domain $D$, where $A=k I \chi_{D}+I \chi_{\Omega / D} \quad(k>0$, $k \neq 1)$. Then, the nontangential maximal function of the gradient of $u$ lies in $L^{2}(\partial D)$.
\end{abstract}

\section{INTRODUCTION}

This paper answers a question that was posed to us by A. Friedman in relation with a better regularity estimate for solutions to certain divergence form equations, which is necessary to prove some theorems of continuous dependence associated to some inverse problems [8].

In particular, we consider the operator $L u=\operatorname{div}(A(X) \nabla u(X))=0$, where $A(X)=k I \chi_{D}(X)+I \chi_{\Omega / D}(X) \quad(k>0, k \neq 1), I$ denotes the identity matrix, $\chi$ is the characteristic function of a set, $\Omega$ is an open set in $\mathbf{R}^{n}$, and $D$ denotes a Lipschitz domain contained in $\Omega$ (see the body of the paper for the relevant definitions). If $u \in W^{1,2}(\Omega)$, the space of square integrable functions in $\Omega$ with distributional derivatives in $L^{2}(\Omega)$, is a weak solution to $L u=0$ in $\Omega$ and the gradient of $u$ has a restriction to the boundary of $D, \partial D$; then the following relation should hold,

$$
k\left\langle\nabla u^{+}(P), N(P)\right\rangle-\left\langle\nabla u^{-}(P), N(P)\right\rangle=0 \text { for } P \text { on } \partial D,
$$

where $\nabla u^{+}, \nabla u^{-}$denote the restrictions of the gradient of $u$ to $\partial D$ from the interior and exterior of $D$ respectively, $N(P)$ denotes the exterior unit normal to $\partial D$, and $\langle\cdot, \cdot\rangle$ denotes the inner product in $\mathbf{R}^{n}$.

In this work we show that under the above conditions, the gradient of $u$ actually has a restriction to $\partial D$ and that the nontangential maximal function of the gradient of $u$ is square integrable on $\partial D$. The main tools used in the proof of this theorem are the invertibility on $L^{2}(\partial D)$ of the operator $\lambda I-K^{*}$,

Received by the editors September 4, 1990 and, in revised form, February 2, 1991.

1991 Mathematics Subject Classification. Primary 35B65; Secondary 35C15, 35P15, 42B20, 45C05, 45E05.

The first author was partially supported by NSF grant DMS $8421377-03$, and the second author was partially supported by NSF grant DMS 8915413. 
where

$$
K^{*}(f)(P)=\text { p.v. } \frac{1}{\omega_{n}} \int_{\partial D} \frac{\langle P-Q, N(P)\rangle}{|P-Q|^{n}} f(Q) d \sigma(Q),
$$

$d \sigma$ denotes surface measure on $\partial D$ and $\lambda$ is a real number with $|\lambda|>\frac{1}{2}$, together with a natural representation of $u$ in a neighborhood of $\bar{D}$ as the sum of a Newtonian potential and a Single Layer potential.

\section{Preliminaries}

The letters $X, Y$ will denote points in $\mathbf{R}^{n}$, and the letters $P, Q$ will denote points of the boundary of a domain $D$ in $\mathbf{R}^{n}$. An open ball of radius $r$ centered at the origin will be denoted as $B_{r}(0)$.

Definitions. A bounded domain $D$ contained in $\mathbf{R}^{n}$ is called a Lipschitz domain if corresponding to each $P \in \partial D$ there is an open, right circular, double truncated cylinder $Z(P, r)$ centered at $P$, with radius equal to $r$, whose basis is at positive distance from $\partial D$, such that there is a rectangular coordinate system for $\mathbf{R}^{n},(x, s), x \in \mathbf{R}^{n-1}, s \in \mathbf{R}$, with $s$-axis containing the axis of $Z$, and a Lipschitz function $\varphi: \mathbf{R}^{n-1} \rightarrow \mathbf{R}$ such that $Z \cap D=\{(x, s): s>$ $\varphi(x)\} \cap Z$, and $P=(0, \varphi(0))$.

By a cone we mean an open, circular, nonempty truncated cone. Assigning one cone $\Gamma(P)$ to each $P$ in $\partial D$, we call the resulting family $\{\Gamma(P): P \in \partial D\}$ regular if there is a finite covering of $\partial D$ by cylinders as in the above definition, such that for each $(Z(P, r), \varphi)$ there are three cones $\alpha, \beta$, and $\gamma$ each with vertex at the origin and axis along the axis of $Z$ such that $\alpha \varsubsetneqq \beta /\{0\} \varsubsetneqq \gamma$ and for all $(x, \varphi(x))=P \in \frac{4}{5} Z \cap \partial D$,

$$
\alpha+P \subset \Gamma(P) \subset \beta+P, \quad \gamma+P \subset Z,
$$

and such that $\left\{\frac{4}{5} Z\right\}$ still covers $\partial D$.

We will need to approximate a given Lipschitz domain $D$, by sequences of $C^{\infty}$ domains $\Omega_{j}, j=1,2, \ldots$, in the manner described in the following lemma $[6,7]$.

Lemma 1. Let $D \subset \mathbf{R}^{n}$ be a bounded Lipschitz domain. Then, the following propositions hold:

(i) There is a regular family of cones $\{\Gamma\}$ as described above.

(ii) There is a sequence of $C^{\infty}$ domains, $\Omega_{j} \subset D$, and homeomorphisms $\Lambda_{j}: \partial D \rightarrow \partial \Omega_{j}$, such that $\sup _{P \in \partial D}\left|\Lambda_{j}(P)-P\right| \rightarrow 0$ as $j \rightarrow \infty$ and for all $j$ and all $P \in \partial D, \Lambda_{j}(P) \in \Gamma(P)$.

(iii) There are positive functions $\omega_{j}: \partial D \rightarrow \mathbf{R}^{+}$bounded away from zero and infinity uniformly in $j$ such that for any measurable set $E \subset \partial D$,

$$
\int_{E} \omega_{j} d \sigma=\int_{\Lambda_{j}(E)} d \sigma_{j}
$$

and $\omega_{j}(P)$ converges pointwise to 1 for a.e. $P$ on $\partial D$. Here, $d \sigma$ and $d \sigma_{j}$ are the surface measures of $\partial D$ and $\partial \Omega_{j}$ respectively.

(iv) The normal vectors to $\Omega_{j}, N\left(\Lambda_{j}(P)\right)$, converge pointwise a.e. to $N(P)$.

(v) There exists a $C^{\infty}$ vector field $\alpha$ in $\mathbf{R}^{n}$ such that for all $j$ and $P \in$ $\partial D\left\langle N\left(\Lambda_{j}(P)\right), \alpha\left(\Lambda_{j}(P)\right)\right\rangle \geq C$, where $C$ depends only on the Lipschitz character of $D$.

The same scheme can be carried out but with $C^{\infty}$ domains containing $D$. 
Definition. Given a function $u$ in $D$ and a regular family of cones $\{\Gamma\}$, we define the nontangential maximal function of $u$ as $u^{*}(P)=\operatorname{Sup}\{|u(X)|: X \in$ $\Gamma(P)\}$ for $P$ on $\partial D$.

We can define a regular family of cones and nontangential maximal functions in $D^{c}$ in a similar manner.

We say that $u$ converges nontangentially a.e. to $f$ if for any regular family of cones $\{\Gamma\}$, we have $\lim _{x \rightarrow P} X \in \Gamma(P) u(X)=f(P)$ for a.e. $P$ on $\partial D$.

Given a Lipschitz domain $D$ in $\mathbf{R}^{n}$, we will denote the Single Layer potential of a function $f \in L^{2}(\partial D)$ as $\mathscr{S}(f)$, where

$$
\begin{aligned}
& \mathscr{S}(f)(X)=\frac{1}{\omega_{n}(2-n)} \int_{\partial D}|X-Q|^{2-n} f(Q) d \sigma, \quad X \in \mathbf{R}^{n}, n \geq 3, \\
& \mathscr{S}(f)(X)=\frac{1}{2 \pi} \int_{\partial D} \log |X-Q| f(Q) d \sigma, \quad n=2,
\end{aligned}
$$

$\omega_{n}=$ surface measure of the unit ball in $\mathbf{R}^{n}$. The following facts are well known.

(i) $\mathscr{S}(f)$ is harmonic on $\mathbf{R}^{n} / \partial D$ and converges nontangentially to $\mathscr{S}(f)=$ $\left.\mathscr{S}(f)\right|_{\partial D}$.

(ii) The gradients of $\mathscr{S}(f)^{+}$and $\mathscr{S}(f)^{-}$have nontangential limits on $\partial D$ when approaching the boundary of $D$ from the interior and exterior respectively [2]. In particular,

$$
\begin{aligned}
& D_{i} \mathscr{S}(f)^{+}(P)=-\frac{1}{2} N^{i}(P) f(P)+K_{i}(f)(P), \\
& D_{i} \mathscr{S}(f)^{-}(P)=+\frac{1}{2} N^{i}(P) f(P)+K_{i}(f)(P),
\end{aligned}
$$

where $N(P)=\left(N^{1}(P), \ldots, N^{n}(P)\right)$ and $K_{i}$ denotes the following principal value operator,

$$
k_{i}(f)(P)=\text { p.v. } \frac{1}{\omega_{n}} \int_{\partial D} \frac{P^{i}-Q^{i}}{|P-Q|^{n}} f(Q) d \sigma(Q) .
$$

In particular,

$$
\left\langle\nabla \mathscr{S}(f)^{ \pm}(P), N(P)\right\rangle=\mp \frac{1}{2} f(P)+K^{*}(f)(P),
$$

where

$$
K^{*}(f)(P)=\text { p.v. } \frac{1}{\omega_{n}} \int_{\partial D} \frac{\langle P-Q, N(P)\rangle}{|P-Q|^{n}} f(Q) d \sigma(Q) .
$$

(iii) $\left\|K_{i}(f)\right\| \leq C\|f\|$, where $C$ depends on the Lipschitz character of $D[1]$, and $\|\cdot\|$ denote the norm in $L^{2}(\partial D)$. 
(iv) $\left\|(\nabla \mathscr{S}(f))^{*}\right\|+\left\|\mathscr{S}(f)^{*}\right\| \leq C\|f\|$, where $C$ depends on the Lipschitz character of $D$ and the regular family of cones [1, $2,7]$.

At this point we have all the necessary ingredients to state the main result of this paper.

Theorem 1. Let $D$ be a bounded Lipschitz domain in $\mathbf{R}^{n} \quad(n \geq 2)$, which is contained in $B_{1 / 2}(0)$. Assume that $u \in W^{1,2}\left(B_{1}(0)\right)$ is a weak solution to $\operatorname{div}(A \nabla u)=0$ in $B_{1}(0)$, where $A(X)=k I \chi_{D}+I \chi_{\mathbf{R}^{n} / D}, k>0, k \neq 1$. Then, the gradient of $u$ has nontangential limits a.e. on $\partial D$ when the boundary is approached from either side and the following estimate holds,

$$
\left\|(\nabla u)^{*}\right\| \leq C\|u\|_{W^{1,2}\left(B_{1}(0)\right)},
$$

where $C$ depends on $k$ and the Lipschitz character of $D$ and the chosen regular family of cones.

To prove this theorem we will use the following result.

Theorem 2. Let $D$ be a bounded Lipschitz domain in $\mathbf{R}^{n} \quad(n \geq 2)$ and $K^{*}$ the operator defined in (1.2). Then for any real number $\lambda$ with $|\lambda|>\frac{1}{2}$, the operator $\lambda I-K^{*}$ is invertible on $L^{2}(\partial D)$.

\section{Proof of Theorem 1}

Let $\Psi$ be a cut-off function supported in $B_{1}(0), \Psi=1$ on $B_{3 / 4}(0)$ and $\Psi=0$ for $|X|>\frac{7}{8}$. From the hypothesis, it follows that $u \Psi \in W^{1,2}\left(\mathbf{R}^{n}\right) \cap$ $C^{\infty}\left(\mathbf{R}^{n} / \partial D\right)$ and $h=\Delta(u \Psi)=u \Delta \Psi+2 \nabla u \nabla \Psi$ on $\mathbf{R}^{n} / \partial D$. In particular, we observe that $h$ is square integrable on $\mathbf{R}^{n}$ with compact support contained in $B_{1}(0)$ and away from $\bar{D}$. Let $v$ denote the Newtonian Potential of $h$,

$$
\begin{aligned}
& v(X)=\frac{1}{\omega_{n}(2-n)} \int_{\mathbf{R}^{n}}|X-Y|^{2-n} h(Y) d Y, \quad X \in \mathbf{R}^{n}, n \geq 3, \\
& v(X)=\frac{1}{2 \pi} \int_{\mathbf{R}^{2}} \log |X-Y| h(Y) d Y, \quad n=2 .
\end{aligned}
$$

For any $f \in L^{2}(\partial D)$, the function $w=u \Psi+v-\mathscr{S}(f)$ is harmonic on $\mathbf{R}^{n} / \partial D$ and lies in $W_{\text {loc }}^{1,2}\left(\mathbf{R}^{n}\right)$. We claim that Theorem 2 implies that one might choose $f$ so that $w$ becomes a weak solution to $\operatorname{div}(A \nabla u)=0$ on the whole $\mathbf{R}^{n}$. With this choice of $f$, the maximum principle for weak solutions to divergence form operators implies that

$$
\sup _{B_{R}(0)}|w| \leq \sup _{\partial B_{R}(0)}|w|,
$$

but for $n \geq 3, w=O\left(|X|^{2-n}\right)$ at infinity. Then $w$ would be identically zero.

Therefore, it only remains to prove the claim. Let $\phi \in C_{0}^{\infty}\left(\mathbf{R}^{n}\right)$ be a test function, and $\Omega_{j}^{+}, \Omega_{j}^{-}$denote two sequences of $C^{\infty}$ domains as in Lemma 1 
such that $\Omega_{j}^{+} \subset D \subset \Omega_{j}^{-}$; then

$$
\begin{aligned}
\int_{\mathbf{R}^{n}} A(X) \nabla w \nabla \phi d X= & \lim _{j \rightarrow \infty}\left(\int_{\Omega_{j}^{+}} k \nabla w \nabla \phi d X+\int_{\mathbf{R}^{n} / \Omega_{j}^{-}} \nabla w \nabla \phi d X\right) \\
= & \lim _{j \rightarrow \infty}\left(\int_{\partial \Omega_{j}^{+}} k\left\langle\nabla w, N_{j}^{+}\right\rangle \phi d \sigma_{j}^{+}-\int_{\partial \Omega_{j}^{-}}\left\langle\nabla w, N_{j}^{-}\right\rangle \phi d \sigma_{j}^{-}\right) \\
= & \lim _{j \rightarrow \infty}\left(\int_{\partial \Omega_{j}^{+}} k\left\langle\nabla u, N_{j}^{+}\right\rangle \phi \Psi d \sigma_{j}^{+}-\int_{\partial \Omega_{j}^{-}}\left\langle\nabla u, N_{j}^{-}\right\rangle \phi \Psi d \sigma_{j}^{-}\right) \\
& +\lim _{j \rightarrow \infty} \int_{\partial \Omega_{j}^{+}} k\left\{\left\langle\nabla v, N_{j}^{+}\right\rangle-\left\langle\nabla \mathscr{S}(f), N_{j}^{+}\right\rangle\right\} \phi d \sigma_{j}^{+} \\
& -\int_{\partial \Omega_{j}^{-}}\left\{\left\langle\nabla v, N_{j}^{-}\right\rangle-\left\langle\nabla \mathscr{S}(f), N_{j}^{-}\right\rangle\right\} \phi d \sigma_{j}^{-} .
\end{aligned}
$$

The first term in the last sum equals

$$
\begin{gathered}
\lim _{j \rightarrow \infty}\left(\int_{\Omega_{j}^{+}} k \nabla u \nabla(\phi \Psi) d X+\int_{B_{1}(0) / \Omega_{j}^{-}} \nabla u \nabla(\phi \Psi) d X\right) \\
=\int_{B_{1}(0)} A(X) \nabla u \nabla(\phi \Psi) d X=0 .
\end{gathered}
$$

From (1.1), Lemma 1, the $L^{2}$ estimate for the nontangential maximal function of the gradient of $\mathscr{S}(f)(1.4)$, and the fact that $v$ is harmonic in a neighborhood of $\bar{D}$, the second limit above is equal to

$$
\int_{\partial D}\left\{(k-1)\langle\nabla v, N\rangle-\frac{k+1}{2} f+(k-1) K^{*}(f)\right\} \phi d \sigma .
$$

Therefore, if $f \in L^{2}(\partial D)$ solves the integral equation $\left(\frac{k+1}{2(k-1)} I-K^{*}\right) f=$ $\langle\nabla v, N\rangle$ on $\partial D$ then the corresponding $w$ satisfies $\operatorname{div}(A \nabla w)=0$ on $\mathbf{R}^{n}$, which proves the claim.

The estimate (1.5) is an easy consequence of (1.4), Theorem 2, and the following estimate,

$$
\|\nabla v\|_{L^{\infty}\left(B_{1 / 2}(0)\right)} \leq C\|u\|_{W^{1,2}\left(B_{1}(0)\right)} .
$$

Remark. When $n=2$ the same argument goes through after one observes the following facts;

(i) The function $h$ has mean value zero on $\mathbf{R}^{n}$.

$$
\begin{aligned}
\int_{\mathbf{R}^{n}} h(Y) d Y & =\int_{B_{1}(0) / D}\{\operatorname{div}(u \nabla \Psi)+\nabla u \nabla \Psi\} d Y \\
& =\int_{B_{1}(0) / D} \nabla u \nabla \Psi d Y=\int_{B_{1}(0)} A \nabla u \nabla \Psi d Y=0 .
\end{aligned}
$$

(ii) Since $v$ is harmonic on a neighborhood of $\bar{D}$, the function $\langle\nabla v, N\rangle$ has mean value zero on $\partial D$. This, together with the fact that $K(1)=\frac{1}{2}$, where $K$ denotes the adjoint operator to $K^{*}$ on $\partial D$ [2], implies that the solution $f$ 
to the above integral equation also has mean value zero on $\partial D$. Therefore, one can show that $w=O\left(|X|^{-1}\right)$ at infinity. This finishes the proof of Theorem 1 .

\section{Proof of Theorem 2}

Let $\lambda$ be a real number with $|\lambda|>\frac{1}{2}$ and set $T f=\left(\lambda I-K^{*}\right)(f)$ for $f$ in $L^{2}(\partial D)$. It was already proved by O. D. Kellogg (Foundations of Potential Theory), that the eigenvalues of $K^{*}$ on $L^{2}(\partial D)$ lie in $\left(-\frac{1}{2}, \frac{1}{2}\right]$ for smooth domains; but his argument also goes through for Lipschitz domains; we will include it here for the sake of completeness.

(i) $T$ is one to one on $L^{2}(\partial D)$. The argument is by contradiction. Suppose that $f \in L^{2}(\partial D)$ satisfies $T f=0$ and $f$ is not identically zero. Since $K(1)=$ $\frac{1}{2}$, it follows by duality that $f$ has mean value zero on $\partial D$. Hence, $\mathscr{S}(f)=$ $0\left(|X|^{1-n}\right)$ and $|\nabla \mathscr{S}(f)|=O\left(|X|^{-n}\right)$ at infinity for $n \geq 2$. Since $f$ is not identically zero, the following numbers cannot be zero,

$$
A=\int_{D}|\nabla \mathscr{S}(f)|^{2} d X \text { and } B=\int_{\mathbf{R}^{n} / D}|\nabla S(f)|^{2} d X .
$$

On the other hand, since $|\nabla \mathscr{S}(f)|^{2}=\frac{1}{2} \Delta\left(\mathscr{S}(f)^{2}\right)$, we have

$$
A=\int_{\partial D}\left(-\frac{1}{2} f+K^{*}\right)(f) \mathscr{S}(f) d \sigma \quad \text { and } \quad B=-\int_{\partial D}\left(\frac{1}{2} f+K^{*}\right)(f) \mathscr{S}(f) d \sigma .
$$

Since $T(f)=0$, it follows that $\lambda=\frac{1}{2} \frac{B-A}{B+A}$. Thus $|\lambda| \leq \frac{1}{2}$, which is a contradiction.

Lemma 2. Let $D$ be a bounded Lipschitz domain in $\mathbf{R}^{n}$ and $\alpha$ denote a smooth vector field on $\mathbf{R}^{n}$ as in Lemma $1(\mathbf{v})$. Then, there exists a constant $C$ depending on $\lambda, n$, and the Lipschitz character of $D$, such that for all $f$ in $L^{2}(\partial D)$ the following estimate holds,

$$
\|f\| \leq C\left\{\left\|\left(\lambda I-K^{*}\right) f\right\|+\|S(f)\|+\|H(f)\|\right\},
$$

where $H$ denotes the following operator on $L^{2}(\partial D)$,

$$
H(f)(P)=\frac{1}{\omega_{n}} \int_{\partial D} \frac{\langle P-Q, \alpha(P)-\alpha(Q)\rangle}{|P-Q|^{n}} f(Q) d \sigma(Q) .
$$

Proof. In what follows, $C$ denotes a constant depending on the Lipschitz character of $D$ and $\lambda$. Let $f \in L^{2}(\partial D)$ and set $u(X)=\mathscr{S}(f)(X)$. From the Rellich-identities [3, 4], we have that

$$
\operatorname{div}\left(\frac{1}{2} \alpha|\nabla u|^{2}\right)=\operatorname{div}(\langle\alpha, \nabla u\rangle \nabla u)+\frac{1}{2}|\nabla u|^{2} \operatorname{div} \alpha-D_{i} \alpha_{j} D_{i} u D_{j} u .
$$

After integrating this identity on $D$, applying Green's formula, and observing that

$$
\begin{gathered}
|\langle\nabla u, N\rangle|=\left|\left(\lambda-\frac{1}{2}\right) f-T(f)\right| \leq|\nabla u|, \\
\langle\alpha, \nabla u\rangle=-\frac{1}{2}\langle\alpha, N\rangle f+K_{\alpha}(f) \quad \text { on } \partial D,
\end{gathered}
$$

where

and

$$
K_{\alpha}(f)(P)=\text { p.v. } \frac{1}{\omega_{n}} \int_{\partial D} \frac{\langle P-Q, \alpha(P)\rangle}{|P-Q|^{n}} f(Q) d \sigma(Q)
$$

$$
\int_{D}|\nabla u|^{2} d X=\int_{\partial D} S(f)\left[\left(\lambda-; \frac{1}{2}\right) f-T(f)\right] d \sigma
$$


we get

$$
\begin{aligned}
\frac{1}{2} \int_{\partial D}\langle\alpha, N\rangle\left(\lambda-\frac{1}{2}\right)^{2} f^{2} d \sigma & \\
\leq & \int_{\partial D}\left[-\frac{1}{2}\langle\alpha, N\rangle f+K_{\alpha}(f)\right]\left[\left(\lambda-\frac{1}{2}\right) f-T(f)\right] d \sigma \\
& +C\|f\|\{\|S(f)\|+\|T(f)\|\}+C\|S(f)\|\|T(f)\| .
\end{aligned}
$$

Multiplying out the integrand in the second integral above and taking to the left-hand side of the inequality the term involving $f^{2}$, we obtain

$$
\begin{aligned}
& \frac{1}{2}\left(\lambda^{2}-\frac{1}{4}\right) \int_{\partial D}\langle\alpha, N\rangle f^{2} d \sigma \\
& \quad \leq\left(\lambda-\frac{1}{2}\right) \int_{\partial D} K_{\alpha}(f) f d \sigma+C\|f\|\{\|S(f)\|+\|T(f)\|\}+C\|S(f)\|\|T(f)\| .
\end{aligned}
$$

If $K_{\alpha}^{*}$ denotes the adjoint operator on $L^{2}(\partial D)$ of the operator $K_{\alpha}$, it is easy to observe that $K_{\alpha}^{*}+K_{\alpha}=H$, where $H$ is the operator defined in Lemma 2 and by duality

$$
\int_{\partial D} K_{\alpha}(f) f d \sigma=\frac{1}{2} \int_{\partial D} H(f) f d \sigma .
$$

Since $|\lambda|>\frac{1}{2}$, the $\|f\|$ can be hidden on the left-hand side of the above inequality, which proves Lemma 2.

Since the operators $S$ and $H$ are compact on $L^{2}(\partial D)$, we conclude from Lemma 2 that $\lambda I-K^{*}$ has closed range.

(ii) If $\lambda$ is real and $|\lambda|>\frac{1}{2}$ then $\lambda I-K^{*}$ is surjective on $L^{2}(\partial D)$ and hence is invertible on $L^{2}(\partial D)$.

Suppose to the contrary that for some $\lambda$ real, $|\lambda|>\frac{1}{2}, \lambda I-K^{*}$ is not invertible on $L^{2}(\partial D)$. Then the intersection of the spectrum of $K^{*}$ and the set $\left\{\lambda \in R:|\lambda|>\frac{1}{2}\right\}$ is not empty and so there exists a real number $\lambda$ that belongs to this intersection and is a boundary point of the set. To reach a contradiction we will show that $\lambda I-K^{*}$ is invertible.

From (i) and Lemma 2 we know that $\lambda I-K^{*}$ is injective and has closed range. Hence there is a constant $C$ such that for all $f \in L^{2}(\partial D)$.

$$
\|f\|_{L^{2}(\partial D)} \leq C\left\|\left(\lambda I-K^{*}\right) f\right\|_{L^{2}(\partial D)} .
$$

Also since $\lambda$ is a boundary point of the spectrum of $K^{*}$ and the real line there exists, a sequence of real numbers $\lambda_{j}$ with $\left|\lambda_{j}\right|>\frac{1}{2}, \lambda_{j} \rightarrow \lambda$, and $\lambda_{j} I-K^{*}$ is invertible on $L^{2}(\partial D)$. In particular given $g \in L^{2}(\partial D)$ there exists unique $f_{j} \in L^{2}(\partial D)$ such that $\left(\lambda I-K^{*}\right) f_{j}=g$.

If $\left\{\left\|f_{j}\right\|\right\}$ has a bounded subsequence then there exists another subsequence that converges weakly to some $f$ in $L^{2}(\partial D)$. Setting $T=\lambda I-K^{*}$, we have

$$
\int_{\partial D} T(f) h d \sigma=\lim _{j \rightarrow \infty} \int_{\partial D} f_{j} T^{*}(h) d \sigma=\lim _{j \rightarrow \infty} \int_{\partial D} T\left(f_{j}\right) h d \sigma=\int_{\partial D} g h d \sigma .
$$

Hence $T f=g$.

In the opposite case we may assume $\left\|f_{j}\right\|=1$ and $\left(\lambda I-K^{*}\right)\left(f_{j}\right)$ converges to zero in $L^{2}(\partial D)$. However from $(*)$

$$
1=\left\|f_{j}\right\| \leq C\left\|\left(\lambda I-K^{*}\right) f_{j}\right\| \leq C\left\|\left(\lambda_{j} I-K^{*}\right) f_{j}\right\|+C\left|\lambda-\lambda_{j}\right| .
$$


Since the final two terms converge to zero as $j \rightarrow \infty$, we arrive at a contradiction. We conclude that for each $\lambda$ real, $|\lambda|>\frac{1}{2}, \lambda I-K^{*}$ is invertible.

The above argument for (ii) is due to Mark Sand, and we thank him for allowing us to use this simplification of our previous proof.

\section{REFERENCES}

1. R. R. Coifman, A. McIntosh, and Y. Meyer, Lintegrale de Cauchy definit un operateau borne sur $L^{2}$ pour les courbes lioschitziennes, Ann. of Math. (2) 116 (1982), 361-387.

2. E. B. Fabes, M. Jodeit, Jr., and N. M. Riviere, Potential techniques for boundary value problems on $C^{1}$ domains, Acta Math. 141 (1978), 165-186.

3. D. S. Jerison and C. E. Kenig, The Neuman problem in Lipschitz domains, Bull. Amer. Math. Soc. 4 (1981), 203-207.

4. J. Neças, Les methodes directes en theorie des equations elliptiques, Academia, Prague, 1967.

5. E. M. Stein, Singular integrals and differentiability properties of functions, Princeton Univ. Press, Princeton, NJ, 1970.

6. G. C. Verchota, Layer potentials and boundary value problems for Laplace's equation in Lipschitz domains, J. Funct. Anal. 59 (1984), 572-611.

7. __ Layer potentials and boundary value problems for Laplace's equation on Lipschitz domains, Thesis, Univ. of Minnesota, June 1982, Appendix.

8. M. Vogelius and A. Friedman, Identification of small inhomogeneities of extreme conductivity by boundary measurements: a theorem on continuous dependence, Arch. Rational Mech. Anal. 105 (1989), 299-326.

Department of Mathematics, University of Chicago, Chicago, Illinois 60637

School of Mathematics, University of Minnesota, Minneapolis, Minnesota 55455

Department of Mathematics, Syracuse University, Syracuse, New York 13244 